\title{
Diagnose Himmel
}

\author{
Eva Jaeggi
}

Psychotherapie-Wissenschaft 8 (1) 11-16 2018

www.psychotherapie-wissenschaft.info

CC BY-NC-ND

DOI: $10.30820 / 8242.01$

\begin{abstract}
Zusammenfassung: Die je unterschiedlichen Vorstellungen der verschiedenen Religionen und Ethnien über das Jenseits («Himmel») können unter psychoanalytischem Gesichtspunkt als unterschiedliche Konfliktlösungen entlang der von der Psychoanalyse herausgearbeiteten Entwicklungskonflikte des Menschen gesehen werden. Diese fantasierten Lösungen sind allerdings nicht wie im irdischen Leben als ein durch das Über-Ich erzwungener Kompromiss zu sehen. Sie sind vielmehr als Wunscherfüllung im Sinne der Unerbittlichkeit des Triebstrebens konzipiert und werden nicht mehr in Frage gestellt. Die sublimierten Aussagen über das Jenseits durch Paulus allerdings sind nicht im Sinne von Entwicklungskonflikten zu interpretieren. Man kann sie eher vergleichen mit den von der relationalen Psychoanalyse und der Humanistischen Psychologie erkannten Wichtigkeit des «Gesehenwerdens» durch den «Anderen».
\end{abstract}

Schlüsselwörter: Psychoanalytische Entwicklungslehre, Kompromiss, relationale Psychoanalyse, humanistische Psychologie

\section{Einleitende Worte}

Wenn ich als Psychoanalytikerin etwas über den Himmel sagen soll, dann muss ich ganz irdisch anfangen. Ich kann bestimmte Vorstellungen vom Himmel, die mir bekannt sind, als Träume und Tagträume auffassen, die etwas verraten können von den Zweifeln, Konflikten und Hoffnungen eines Menschen.

Ich kann sie natürlich auch als Utopien annehmen doch in dieser Kategorie kommt der Himmel bei vielen Soziologen und Philosophen schlecht weg. Er wird dann als eine Täuschung des Volkes angesehen, die verhindern soll, dass das Volk sich den realen Zuständen und ihren möglichen Veränderungen zuwendet angesichts der utopischen Verheissungen, die das Leben nach dem Tod bietet.

Ich möchte mich aber nicht unbedingt damit beschäftigen. Bleiben wir beim Träumen.

\section{Diagnostische Varianten}

Wenn man als Psychoanalytikerin die Träume zu Hilfe nimmt, um Menschen besser zu verstehen, dann orientiert man sich nicht an Krankheitsvorgaben oder diagnostischen Kategorien wie wir sie in verschiedenen Persönlichkeitstheorien vorfinden. Psychodynamischer Traumtheorie zufolge werden in Traumbildern Lebensthemen, Konfliktfelder und dominierende Wünsche verarbeitet - seien sie nun unbewusst oder auch schon mehr oder weniger bewusstseinsfähig. Man muss also immer wieder die ganze Person, ihr persönliches und gesellschaftliches Umfeld bedenken, wenn man einen Traum «diagnostiziert»wobei dieses Wort im psychoanalytischen Umfeld nicht dieselbe Bedeutung hat wie in der Medizin. Es gibt dabei keine vorgeformten Bedeutungen à la «eine schwarze
Katze bedeutet Unglück». Die innere, jeweils in persönlichkeitsspezifische Bilder gepackte «Botschaft» eines Traumes wird erst dann entdeckt, wenn der Träumende seine eigenen Assoziationen zum Traum einbringt.

Es bleibt daher immer ein Wagnis, wenn man versucht, mit psychoanalytischen Kategorien ein kulturelles Gebilde - sei es ein Kunstwerk, sei es ein kollektiver Traum wie der vom Himmel - zu «diagnostizieren». Man hat aber immerhin eine Menge an Daten aus dem gesellschaftlichen Umfeld eines kulturellen Gebildes, um daraus Hypothesen ableiten zu können. Es ist den Versuch wert, aus solchen Kollektivträumen die aus individueller Beobachtung gewonnenen «typischen» Problemfelder, Wünsche und Abwehrbewegungen des Kollektivs, dem diese Träume entstammen, herauszulesen.

Nicht in jeder Kultur aber sind gerade die Vorstellungen vom Jenseits Thema sehr spezifischer Überlegungen. Vorhistorische oder auch manche antike Religionen haben zwar Vorstellungen vom Jenseits, aber in vielen Kulturen wird das Jenseits nicht unbedingt in solch prägnanten Bildern vorgestellt wie im Christentum und in den muslimischen Konfessionen. Grabbeigaben in antiken Gräbern lassen darauf schliessen, dass das jenseitige Leben sich nicht allzu sehr vom irdischen unterscheidet - so bei den Ägyptern oder bei manchen Stämmen der Germanen.

Das Judentum spricht in verschlüsselten Worten von einer spirituellen Ganzheit, die der Seele nahe bei Gott unendlichen Frieden verleiht. Manche jüdische Autoren konzentrieren sich eher auf die Auferstehung, die mit der Ankunft des Messias verbunden ist. Es entsteht dann ein neues, besseres Leben im irdischen Dasein. Bei den meisten jüdischen Autoren bleiben die Vorstellungen aber eher abstrakt. Wichtiger ist das irdische Leben, in dem man möglichst viel Gutes tun soll.

Wenn man aber im christlichen Kulturkreis aufgewach- 
sen ist, verknüpft man mit dem Wort «Himmel» (sofern man nicht Astronom ist) unzählige Bilder von geflügelten Engeln, Wolken, Musikinstrumenten, hellen, farbenfrohen Gewändern und selig lächelnden Menschen. In vielen, vielen Kirchen kann man dies alles auf grossen Bildtafeln und Gemälden bestaunen. Auch Gott selbst lächelt oft gütig, manchmal streng von seinem Thron herab. Wurde man in einer noch kindlich-religiösen Form erzogen, so hat man solche Vorstellungen meist verbunden mit ewig glücklichem Spielen ohne jeden Zwang, mit Blumen, schönen Früchten und Musik.

Auch der Islam kennt wunderbare Bilder, allerdings werden diese Bilder entsprechend dem Darstellungsverbot nur verbal überliefert.

Meine Aufzählung der verschiedenen Himmelsbilder ist natürlich nicht vollständig, ich bin weder Theologin noch Religionswissenschaftlerin, kann also diese Vorstellungen auch nicht jederzeit ganz sauber einzelnen Religionen oder Zeitabschnitten zuordnen. Es geht mir auch vielmehr darum, diese Bilder mit allgemein menschlichen Wünschen und Konflikten zu verbinden - so wie man auch in der Psychoanalyse Träume mit solchen Wünschen verbindet. Sie sind ubiquitär. Man kann also auch die Himmelsvorstellungen als den Versuch, menschliche Konflikte darzustellen und zu lösen, ansehen. Die Dreigliederung in orale, anale und ödipale Entwicklungsstufen entspricht dem, was wir bei jedem Menschen wahrnehmen können: Eine dieser Stufen mag im Vordergrund stehen oder vielleicht im Sinne nicht ganz klarer Lösung ambivalent besetzt sein. In der Himmelsvorstellung allerdings ist jeder Wunsch erfüllt.

\section{Paradies und Schlaraffenland - die Oralität dominiert}

Der Himmel ist eine wunderbare Stadt (z.B. das himmlische Jerusalem), voll der kostbarsten Baumaterialien, umgeben von der schönsten Landschaft, die Räume sind ausgestattet mit Edelsteinen, es herrscht keine Not.

Der Himmel in den islamischen Räumen ist ein $\mathrm{Ru}$ heraum mit vielen Gemächern, ebenfalls kostbar ausgestattet. Das herrlichste Essen wird serviert, die berühmten Jungfrauen bedienen die Männer, vor allem die Märtyrer. Wichtig dabei ist, dass des Märtyrers Manneskraft nie versiegt, auch wenn es 77 Jungfrauen sind, die mit ihm in einer Abfolge verkehren.

Die «ewigen Jagdgründe» dürften den Indianern genau das geboten haben, was ihnen im irdischen Leben wichtig ist: genügend Fleisch und vielleicht auch das Vergnügen am Jagen - wobei wohl der Erfolg garantiert ist.

Mit einem Wort: Es ist alles da, und dies ohne dass man etwas dafür tun muss. Der Himmel bietet alles umsonst: Versorgung pur.

\section{Fliegen-können - die Analität dominiert}

Ein anderer, eher esoterischer Aspekt: die Weite, das Fliegen, hinauffliegen, Flügel haben. Eine mir bekannte Künstlerin, die um ihren nahen Tod wusste, schrieb ein paar Tage vor ihrem Tod an alle ihre Freunde und Bekannten eine E-Mail-Botschaft (die ihre Familie gleich nach ihrem Tod absandte): «Ich habe mich auf eine Reise begeben, ohne Gepäck, es ist wunderbar, so leicht zu fliegen.» Alle populären Kindervorstellungen vom Himmel spielen mit diesem Gedanken: auf Wolken zu sitzen, ganz leicht zu sein, nicht der Schwerkraft unterworfen, ein Engel zu sein. Dies ist Autarkie pur - nichts und niemand behindert die frei fliegende Seele.

\section{Liebe - Ödipalität dominiert}

Dass wir geliebt werden im Himmel, alle unsere Lieben wiedersehen und jeder Konflikt schweigt - auch das gehört zu unseren Vorstellungen vom Himmel. Meist sind sie nicht sehr bilderträchtig, eher Beschreibungen seelischer Zustände. Ich erinnere allerdings einen Film, den ich in Utah bei den Mormonen gesehen habe (das war Anfang der 70er Jahre, er ist, glaube ich, jetzt nicht mehr zu sehen), da war das liebende Zueinanderkommen der Familien bildlich dargestellt: Der Grossvater (im weissen Nachthemd) schwebt der Oma und seinen eigenen Eltern sowie frühverstorbenen Kindern - alle im Nachthemd glückselig entgegen. Das mag man komisch finden, ist es auch, aber wie soll man sich konkret diese problemlose Liebesgemeinschaft vorstellen? Unsere Bilder sind ja immer kindlich-konkret, oft archaisch geprägt, wenn es um wichtige Themen geht.

\section{Das Jüngste Gericht und die Hölle}

Und dann wäre da ja auch noch das Jüngste Gericht meist ein eher schreckenerregendes Bild, wenn wir uns die verschiedenen Darstellungen der Hölle vor Augen halten. Meistens sind sie vom Gegenteil des Himmels dominiert: leere Räume, ewige Qualen, schreckliche Einsamkeit und grausames Festgehaltenwerden. All dies ist das Gegenbild des Himmels, sozusagen die andere Seite der Konflikte, die auf diesen drei Entwicklungsstufen möglich sind.

\section{Das Schauen Gottes}

Die sehr ernsthaften theologisch besonders bedeutsamen Versuche über den Himmel aber beschäftigen sich mit dem wichtigsten Aspekt des Himmels: mit der Anschauung Gottes, wobei mir schon in meiner Kindheit des Augustinus' Aussagen zum glückseligen «Schauen Gottes» am meisten Eindruck gemacht hat. Ich habe daher auch auf die Todesanzeige meiner Mutter den Satz geschrieben: «Sie darf nun schauen, was sie geglaubt hat», worauf eine meiner atheistischen Freundinnen spöttisch sagte, als sie dies las: «Na, die wird aber schauen ...»

Auf die vermutlich tiefste diesbezügliche Stelle im ersten Paulusbrief an die Korinther werde ich noch einmal genauer hierauf zu sprechen kommen.

\section{Erklärungen/Hypothesen}

Wenn man die Analogie zur Freud'schen Entwicklungslehre weiter betreibt und den Himmel als ein Traumgebilde diagnostiziert, dann ergeben sich in den unterschiedlichen Jenseitsvorstellungen Varianten der bedeutenden Kernkonflikte, die man auch im menschlichen Leben vorfindet. So wie es Menschen gibt, deren Sehnsucht 
nach Versorgung einen zentralen Teil ihrer Wünsche (und dem entsprechend auch ihrer Konflikte) bildet, so gibt es auch Menschen, denen persönliche Autonomie oder auch Probleme von Liebe und Eifersucht das wichtigste Lebensthema darstellen.

\section{Das Schlaraffenland: Der orale Himmel}

Man kann sich Menschen vorstellen, die von übersteigerten irdischen Gütern träumen: Alles ist schon da, man wird verwöhnt, Essen, Trinken - die Nähe zum Schlaraffenland ist offensichtlich -, die wunderschöne Umgebung: Man bekommt alles geschenkt. Was würde ich aus solcherart Träumen für einen eben ganz irdischen Menschen mutmassen, in welcher Richtung würde ich (wenn er ein Patient ist) versuchen, in der Therapie mit ihm zu denken? Ich würde an einen sehr grundlegenden Konflikt denken, der dieses Sehnsuchtsbild hervorbringt. Dieser Konflikt heisst in der Sprache Freuds «der orale Konflikt», in moderner Sprache sagen wir «VersorgungsAutarkie-Konflikt».

Was ist damit gemeint? Ich gehe davon aus, dass jemand der sich solche sehr konkretistischen Vorstellungen von einem Paradies erträumt (vor allem, wenn er es sehr oft tut) ein zentrales Ungenügen in sich spürt: das Gefühl, man werde nicht genügend versorgt, es werde einem nichts geschenkt, man müsse sich alles erringen. Dies wird dann konterkariert (sonst wäre es kein Konflikt) von dem Bestreben, alles für andere tun zu müssen, sich eben alles «erringen» zu müssen: Liebe, Erfolg, materielle Güter.

Es gibt zwei Möglichkeiten, wie dieser Konflikt - sofern er zentral ist - gelebt werden kann: Es kann sich um Menschen handeln, die andere hemmungslos ausnützen, um versorgt zu werden. Jede Art Schmarotzer fällt darunter, alles Klagen darüber, wie ungerecht das Leben einen behandelt hat etc. etc. Das nennt man dann die regressive Variante, in der der innere Konflikt agiert und gelebt wird. Die andere, die progressive Variante lebt derjenige, der alles für andere tut, nicht «nein» sagen kann, wie die moderne Vokabel heisst, das heisst sich ausnützen lässt. Es ist die andere Seite der Medaille. Dass es sich dabei um einen Konflikt handelt, merkt man daran, dass nicht nur derjenige, der sich ungerecht behandelt fühlt und alles «geschenkt» haben will, unglücklich ist; sondern dass auch derjenige, der alles für andere tut, damit keineswegs zufrieden ist. Er ist dauernd überfordert, nervös, hat alle möglichen Erschöpfungssymptome und depressive Verstimmungen. Auch für ihn ist die Welt, in der man nichts geben muss, sich einfach nur verwöhnen lässt - mit Essen, mit Schönheit, mit Sex (was immer auch ein Mensch für erstrebenswert hält), das Ideal. Beide Typen mit diesem Versorgungskonflikt haben also keinen Ausgleich gefunden zwischen den basalen Strebungen jedes Menschen: sich versorgen zu lassen und andere zu versorgen - je nach Umständen.

Der Traum vom endlich ganz und gar Versorgtwerden ist also der Traum dessen, der noch nicht akzeptiert hat, dass zwischen den beiden Strebungen des Versorgens und des Sich-versorgen-Lassens eine Balance gefunden werden muss. Es ist also nicht nur der Traum desjenigen, der zu wenig hat und aus der äusseren Not heraus sich ein Schlaraffenland erträumt. Es ist der innere Konflikt, der im Traum im Sinne der Wunscherfüllung gelöst wird. Eine Wunscherfüllung, auf die allerdings ein schlimmes Erwachen erfolgen kann - wenn er in der Hölle desjenigen landet, dem kein Wunsch mehr erfüllt wird, der in der Hölle dursten und hungern muss.

\section{Ihr seid frei: Der anale Himmel}

Sehen wir uns die oft etwas naiv-esoterischen kindlichen Himmelsvorstellungen an. Diese werden übrigens real sehr oft geträumt: fliegen, sich den irdischen Hemmungen der Schwerkraft nicht unterziehen müssen usw. Manchmal fällt man dann unsanft aus dem Bett oder erwacht zumindest fallend.

Was könnte dahinterstecken?

Wir kennen in der Psychoanalyse noch einen weiteren wichtigen Konfliktherd: das Streben nach individueller Autonomie versus Abhängigkeit. Auch dieses Bestreben ist ubiquitär, muss nicht konflikthaft sein, aber in solchen (wiederholten) Träumen zeigt sich eben die Auseinandersetzung mit einer bestehenden Konfliktsituation. Jeder Mensch (wie die meisten Tiere auch) hat schon in jüngsten Lebenstagen das Bedürfnis, sich ein klein wenig Autonomie zu bewahren - und sei es nur, dass der Säugling instinktiv den Kopf von der Brust wegdreht, wenn er nicht mehr trinken will. Es gibt aber dazu ebenfalls ein Pendant: das Bestreben, sich von anderen leiten und beschützen zu lassen, wichtige Menschen nahe bei sich zu haben. Wir wissen und spüren, dass wir abhängig sind - jeder Mensch! -, aber gleichzeitig wollen wir uns auch unsere Unabhängigkeit bewahren, etwas ganz Eigenes sein, unseren Willen durchsetzen. Das kann bei manchen Menschen zum Konflikt werden. Auch dieser Konflikt kann regressiv oder progressiv gelebt werden. Es gibt Menschen, die sich alleine nichts zumuten, immer abhängig bleiben und solche, die jede Abhängigkeit leugnen, lieber andere von sich abhängig machen als selbst abhängig zu sein. Wenn dieser Wesenszug auch noch gepaart ist mit einer gehörigen Portion Selbstbezogenheit, also Narzissmus, dann finden wir die merkwürdigsten Verhaltensweisen: sich grossen Gefahren aussetzen, zu leugnen, dass man auch von äusseren Mächten abhängig ist etc. - daher das «Fliegenkönnen». Dass wir daraus selten ohne das «Herunterfallen» erwachen ist Ausdruck der Enttäuschung, die wohl auch Ikarus gehabt hat, als er merkte, dass er nicht zur Sonne hinauffliegen kann. Die Schwerkraft überwinden, einen schwerelosen Körper zu haben - das wäre die perfekte Autonomie. Auch hier finden wir in der Hölle das Gegenbild: Man wird gefesselt, kann nicht entfliehen, ist den teuflischen Monstern ausgeliefert.

\section{Heilwerden: Der ödipale Himmel}

Die Vorstellung von der «allgemeinen Liebe», von der konfliktfreien Begegnung mit allen, die uns wichtig sind, vor allem auch mit unserer Familie, zielt dann auf noch einen wichtigen Konfliktherd: dem Bedürfnis einerseits intime zum Beispiel familiäre Beziehungen frei zu halten von Eifersucht, Neid und Aggression und andererseits dem Bedürfnis, sich gegen Konkurrenten durchzusetzen, 
der Wichtigste zu sein, geliebt zu werden ohne Abstriche machen zu müssen.

In konkreten Träumen kann sich das in vielerlei Bildern ausdrücken - das ist natürlich sehr abhängig von der jeweiligen individuellen Situation, wo dann konkrete Bekannte oder Familienmitglieder den Traum bevölkern. Als Jenseitsvorstellung ist es zum Beispiel der Hades der Antike. Als christliche Jenseitsvorstellung gibt es sehr allgemeine Aussagen über die «Liebes- und Festgemeinschaft». Es gibt zwar meist nicht dieselben kräftigen Bilder wie diejenigen, die die äusseren Herrlichkeiten des himmlischen Paradieses beschreiben, aber es sind Gedanken von Liebe und Harmonie und konfliktfreiem Zusammenleben.

Christliche Theologie hat sich recht häufig um diese Vorstellung der endlich vereinigten Menschen Gedanken gemacht. Es sind vor allem Vorstellungen, dass man - wie bei Paulus (vgl. hierzu Lang \& McDannell, 1988) - keine alten menschlichen Bindungen mehr spürt, und auch solche - wie bei Augustinus -, dass wir durchaus lieben wie im irdischen Leben - nur besser und übrigens ohne Sexualität, die ja bekanntlich sehr oft Probleme mit sich bringt:

\begin{abstract}
«Unsere Lieben, die dieses Leben verlassen, sind uns nicht verloren; wir haben sie nur vorausgeschickt. Aufgrund der festen Verheißung dürfen auch wir hoffen, einst in jenes Leben einzutreten, in welchem wir sie umso mehr lieben werden, je besser wir sie kennen und wo wir sie lieben werden ohne Furcht vor dem Scheiden» (Augustinus, 397-401 [1955]); zit.n. Lang \& McDannell, 1988, S. 399).
\end{abstract}

Als individuelles Traumbild finden wir immer wieder das «Verlassenwerden» und das «Wiederfinden" geliebter Personen. Die naiven Vorstellungen davon, dass uns auf der Himmelstreppe alle unsere verstorbenen Lieben in einem nunmehr ewigen Beisammensein entgegen schweben, sind das theologische Pendant dazu. Man wird im Jenseits geliebt ohne Bedingungen. Der Konflikt zwischen Liebe und Aggression soll so überwunden werden.

\section{Jenseits von Gut und Böse: Der schuldfreie Himmel}

Das «Jüngste Gericht» ist natürlich in sehr vielen Träumen irgendwie vorhanden. Was sich dahinter verbirgt, ist nicht schwer zu erschliessen. Es sind unsere Schuldgefühle - reale wie auch irreale, die uns in vielen berühmten Darstellungen zeigen, wie wir belohnt oder bestraft werden können. Es ist wie auch im richtigen Leben: Das Strafgericht ist konkreter und lebendiger als die Belohnung, was wir schon in Dante's Reise durch das Jenseits erfahren können. Der Himmel ist bekanntlich immer ein wenig fade in den vielen Darstellungen der Kunst. Das Gefühl, man sei «irgendwie» schuldig geworden, überwiegt, im Leben wie in der Therapie. Es durchzieht alle oben genannten Konflikte

\section{Trost auf höherer Ebene -}

die relationale Psychotherapie und der heilende Dialog.

Kommen wir zu den theologisch sehr relevanten eher abstrakten Aussagen über den Himmel, die «seligma- chende Gottesschau» des Augustinus und des noch viel ausdrucksstärkeren Schauen Gottes, wie Paulus es ausdrückt, nämlich das «Erkanntwerden.»

Bei Augustinus ist diese Anschauung Gottes mit sehr viel Glück verbunden: «Dann werden wir Muße haben und schauen, schauen und lieben und loben» (zit.n. Lang \& McDannell, 1988). Es ist die mystische Vereinigung mit Gott, die Augustinus selbst als ungeheuer glücklich empfunden zu haben scheint, als er immer wieder mystische Erfahrungen machte.

Hier aber endet die Analogie mit unserer profanen bildhaften Traumwelt.

Das mystische Erlebnis gehört natürlich vorerst in die Welt der Theologie, wo es seinen alten und festen Standplatz hat und von den Mystikern in vielen Epochen sogar als ein Wahrheitsbeweis für die Wirklichkeit Gottes angesehen wurde.

Man kann hieran natürlich auch mit psychologischen Kategorien herangehen und diese beseligende Schau Gottes, die wir im Himmel haben sollen, als eine Sehnsucht nach Verschmelzung ansehen, die uns auch im irdischen Leben begegnet - und sowohl Angst als auch Entzücken bereiten kann. Aus der Verschmelzung aufzutauchen (sei es diejenige der Sexualität, der innigen geistigen Übereinstimmung, des gefühlsmässigen In-eins-Seins oder das Entzücken über Kunst und Natur): All dies steht unter der Drohung des Auseinandergehens, des Abschieds, des Zurückgelassenwerdens in eine Welt, in der man wieder ganz alleine ist. Dieser Abschied aber heisst: merken, dass man alleine ist. Diese universale Einsamkeit gibt es nach Augustinus im Jenseits nicht mehr, so wie es übrigens auch nicht mehr die ausschliessenden Familienbindungen geben soll. Keiner ist mehr ein Fremder. Auch die Vorstellung von Paulus, dass wir alte Bindungen abgelegt haben, befreit uns vor dem Abschiedsschmerz.

Was aber können wir im berühmten Paulus-Wort als psychologisch Denkende erkennen? Es heisst im ersten Korintherbrief:

«Wir schauen jetzt im Spiegel rätselhaft; dann aber von Angesicht zu Angesicht. Jetzt erkenne ich bruchstückweise, dann aber werde ich klar erkennen wie auch ich bin erkannt worden. Jetzt aber bleiben Glaube, Hoffnung und Liebe diese Drei. Das Grösste unter ihnen aber ist die Liebe» (1. Kor 13, 12-13).

Ich glaube, dass hier von einem Mann der Antike das tiefste Verständnis für das menschliche Leben ausgesprochen wird. Es ist das Verständnis dafür, dass unser Erkenntnisstreben nie ruhen kann («immer Stückwerk bleibt») und wir nur in der unfassbaren Zeitlosigkeit der Ewigkeit darauf hoffen können, das Ziel vieler Philosophen zu erreichen, nämlich: das Ganze zu erkennen, wobei eben die eigene Person mit eingeschlossen ist in diese Erkenntnis.

Erkenntnis des Allgemeinen und Selbsterkenntnis fallen also bei Paulus zusammen. Nur wer sich selbst so sehen kann, wie Gott ihn sieht, kann auch das "Andere» erkennen. Die Selbsterkenntnis aber ist nur möglich durch einen anderen. Die anderen Menschen «sehen» uns al- 
lerdings nur in unseren jeweiligen Teilaspekten. Selbst in der Liebe sind es nur «Bruchstücke», die wir im anderen erkennen. In der Zeitlosigkeit Gottes aber wird - so der Traum von Paulus - jeder Einzelne als Ganzes liebend erkannt. Nur die Liebe Gottes, die umfassender ist als die menschliche Liebe, kann dies bewirken. Die Hoffnung aller Menschen, geliebt zu werden in allem, was sie sind - eine im Diesseits nie zu verwirklichende Hoffnung -, findet hier ihre Erfüllung.

In der relationalen Psychoanalyse (sowie in der Humanistischen Psychologie) wird dieses Moment im therapeutischen Dialog sehr stark betont. Es ist das "Gesehenwerden», das verbunden ist mit einem «Akzeptiertwerden», das dem Menschen Heilung und Befriedung bringen soll. Der «Andere» mit seinem erkennenden Blick ist nötig, um den Menschen aus seiner Selbstbezogenheit zu befreien. In dieser Befreiung erkennt er aber auch den anderen. Den anderen (das «Andere») als etwas Getrenntes zu erkennen, bedeutet: zu sehen, dass die absolute Verschmelzung nicht möglich ist, dass aber durch die Liebe dieses schmerzliche Erkennen gemildert werden kann. Wir als Psychologen können nur von Linderung sprechen - keine Therapie, auch keine Liebe, kann vollkommen trösten. Dem Theologen aber sind vielleicht noch andere Verheissungen möglich: Es ist der Glaube und die Hoffnung auf vollkommene Heilung.

\section{Zum Abschluss}

Ich habe den Himmelsträumen sehr oft die basalen menschlichen Konflikte zugrunde gelegt. Sie werden in den Träumen vom Schlaraffenhimmel, vom Fliegen und von der ewigen und konfliktfreien Liebe immer zugunsten der - im Psychoanalytiker-Jargon würde man sagen regressiven Lösung geträumt. Alles ist «Friede, Freude, Eierkuchen» könnte man ganz profan sagen.

Das Paulus-Wort aber (und ich könnte ihm keinen Bildertraum entgegensetzen? Vielleicht eine künstlerische Darstellung anderer Art? Abstrakt? Minimalistisch?) berührt in ganz anderer Weise. Für mich deute ich es so: Hier werden die letztlich unlösbaren Konflikte, denen Menschen immer unterworfen sind, nicht einseitig gelöst. Sie werden in aller Tiefe erkannt - wie Paulus sagt: von Gott. Das heisst: Sie werden in Liebe erkannt und wir Menschen sehen sie deshalb auch in aller Tiefe, weil sie eben die Liebe eines Höheren zeigt. Es ist sozusagen die dritte Stufe in der Dialektik des Erkennens. Dass wir hin- und hergerissen sind zwischen jeweils zwei Polen, sozusagen die erste und zweite Stufe der Erkenntnis, (Sorgen/Versorgtwerden; Freiheit/Gebundenheit; Lieben/Hassen) - diese Erkenntnis ist nur «Stückwerk. Es kann aber die dritte Stufe der Erkenntnis geben, die diese Pole in sich einschliesst. Wie dies aussieht, was wir dann sehen und erleben - das übersteigt wohl unsere diesseitige Erkenntnismöglichkeit. In unserem beschränkten Erkenntnisvermögen können wir nur davon sprechen, dass wir «eine Balance» halten wollen, dass es «immer Kompromisse geben muss» zwischen unseren ambivalenten Bestrebungen. Vielleicht aber gibt es eine ganz andere Möglichkeit diese Konflikte zu erfahren: die Überwindung aller Gegensätze, die im Menschen solche Unruhe hervorrufen, bedeutet vielleicht eine neue Form des Existierens, in der es solche Gegensätze, Ambivalenzen und Konflikte nicht mehr geben muss. Vielleicht ist das Nirwana der Buddhisten in dieser Weise zu verstehen?

Wir als Christen können, so das Paulus-Wort, nur ahnen, dass es diese Möglichkeit der Konfliktfreiheit gibt - vielleicht durch ein «ganz Anderes», das man Gott nennen kann. Es gibt die Aufhebung der Gegensätze in einem Dasein, das diese Gegensätze überwunden hat und nicht wieder ein Ansatzpunkt für neue Gegensätze werden kann. Wenn wir in diesem Zusammenhang von liebender Erkenntnis sprechen, dann muss auch an Versöhnung und Vergebung gedacht werden. Versöhnung mit uns selbst und unseren Konflikten sowie Versöhnung und Vergebung mit und für den anderen. Welche Form der Liebe aber dieses versöhnende Erkennen ermöglicht - das kann ich als Psychoanalytikerin natürlich nur als eine offene Frage formulieren.

\section{Literatur}

Altmeyer, M. \& Thomä, H. (2006). Die vernetzte Seele. Stuttgart: Klett-Cotta.

Augustinus (397-401 [1955]). Confessiones - Bekenntnisse (Übers. Joseph Bernhard). München: Kösel.

Die Heilige Schrift des alten und des neuen Bundes (1958). Übers. von Paul Riessler und Rupert Storr. Mainz: Matthias GrünewaldVerlag.

Lang, B. \& McDannell, C. (1988). Der Himmel. Eine Kulturgeschichte des ewigen Lebens. Frankfurt a. M.: Insel-Verlag.

Mertens, W. (1994). Entwicklung der Psychosexualität und der Geschlechtsidentität, $B d$. 2. Stuttgart: Kohlhammer.

\section{Diagnosis Heaven}

The psychoanalytical perspective may view the different perceptions of the various religions and ethnicities about the afterlife (heaven) as different solutions to conflicts in line with the construct of man's developmental conflicts that have been identified by psychoanalysis. However, these fantasized solutions should not be regarded as a compromise imposed by the superego, as is the case with the earthly life. Instead, they were designed as a wish fulfilment in the sense of the relentlessness of the instinctual drive and are no longer called into question. The sublimated statements about the afterlife by Paul, however, are not to be interpreted as developmental conflicts. They are more comparable to the importance of «being seen» by the «other» as recognised by intersubjective psychoanalysis and humanistic psychology.

Keywords: Psychoanalytic Developmental Theory, Compromise, Intersubjective Psychoanalysis, Humanistic Psychology

\section{Diagnosi Cielo}

Le differenti rappresentazioni delle diverse religioni ed etnie sull’al di là («Cielo») possono essere viste dalla prospettiva psicoanalitica come diverse soluzioni al conflitto nel corso dei 
conflitti dello sviluppo dell'uomo. Queste soluzioni di fantasia nella vita terrena non sono comunque da vedere come un compromesso condizionato dal super-io. Sono piuttosto concepite come un adempimento dei desideri nei confronti dell'implacabilità dello sforzo dell'istinto e non vengono più messe in discussione. Le asserzioni sublimate sull'al di là da parte di Paolo non sono comunque da interpretare nel senso di conflitti di sviluppo. Possiamo paragonarli alla riconosciuta importanza da parte della psicanalisi relazionale e della psicologia umanistica dell' «essere visti» da parte degli «altri».

Parole chiave: teoria evoluzionista psicoanalitica, compromesso, psicanalisi relazionale, psicologia umanistica

\section{Die Autorin}

Eva Jaeggi, em. Prof. für Klinische Psychologie, ist Psychoanalytikerin und Verhaltenstherapeutin, darüber hinaus Autorin zahlreicher Bücher und Artikel (u.a. Vergleich von Therapieschulen, Moderne Lebensformen, Beruf des Therapeuten, Alter).

\section{Kontakt}

Eva.Jaeggi@tu-berlin.de 